\title{
Comprensión lectora en estudiantes de escuela preparatoria abierta: efecto de una intervención basada en la motivación y las estrategias metacognitivas
}

\section{Reading Comprehension in Open High School Students: Effect of a Motivation Based Intervention and metacognitive strategies}

\author{
Otoniel Ruvalcabar-Estrada, ${ }^{1 \mathrm{a}}$ Jorge A. Hilt ${ }^{2}$ y Jorge Omar Trisca ${ }^{3}$ \\ Universidad de Montemorelos, Nuevo León, México ${ }^{123}$ \\ iD Orcid ID: https://orcid.org/0000-0003-1406-9618 ${ }^{1}$ \\ (iD) Orcid ID: https://orcid.org/0000-0002-5860-298212 \\ (iD) Orcid ID: https://orcid.org/0000-0002-0712-7042 3
}

Recibido: 27 de enero de 2021

Aceptado: 28 de marzo de 2021

\begin{abstract}
Resumen
El propósito de este estudio fue determinar si existe una diferencia significativa del nivel de comprensión lectora entre un pretest y un postest, en alumnos de una escuela preparatoria abierta de Nuevo León (México) después de una intervención basada en la motivación y las estrategias metacognitivas. Se utilizó un diseño cuantitativo cuasi experimental con una intervención de 14 semanas entre la aplicación de ambas mediciones. La población estuvo compuesta por 18 alumnos a quienes se les aplicaron tres instrumentos, el Test de Cloze para medir la comprensión lectora, la Escala Atribucional de Motivación de Logro General y el Inventario de Estrategias Metacognitivas Generales. Los hallazgos mostraron que existe una diferencia significativa de comprensión lectora $(\mathrm{t}(17)=3.653, \mathrm{p}=.002$, Cohen's $\mathrm{d}=0.86) \mathrm{y}$ estrategias metacognitivas $(\mathrm{W}=30.00, \mathrm{p}=.017$, Correlación biserial $\mathrm{r}=0.649$ ) entre el pretest y el postest, aunque no fue significativo en el nivel de motivación $(\mathrm{t}(17)=-0.341$, p $=.737$, Cohen's d = 0.008). En conclusión, mediante la ejecución de un programa de intervención en estrategias metacognitivas y motivación hacia la lectura, se obtuvo una mejora en el nivel de comprensión lectora de los participantes.
\end{abstract}

Palabras clave: Comprensión lectora; cognición; motivación de logro; intervención

\footnotetext{
Abstract

${ }^{\mathrm{a} C}$ Correspondencia a los autores:

E.mail: otonielruval@hotmail.com; jorgehilt@um.edu.mx; trisca@um.edu.mx
}

The purpose of this study was to determine if there is a significant difference in the level of reading comprehension between a pretest and a posttest, in students of an open high school in Nuevo León (Mexico) after an intervention based on motivation and metacognitive 
strategies. A quasi-experimental quantitative design was used with an intervention of 14 weeks between the application of both measurements. The sample consisted of 18 students to whom three instruments were applied, the Cloze Test to measure reading comprehension, the Attributional Motivation Scale of General Achievement and the Inventory of General Metacognitive Strategies. The findings showed that there is a significant difference in reading comprehension $(\mathrm{t}(17)=3.653, \mathrm{p}=.002$, Cohen'sd $=0.86)$ and metacognitive strategies $(\mathrm{W}$ $=30.00, \mathrm{p}=.017$, Biserial correlation $\mathrm{r}=0.649$ ) between the pretest and the posttest, although it was not significant in the level of motivation $(t(17)=-0.341, p=.737$, Cohen'sd $=0.008)$. In conclusion, through the execution of an intervention program in metacognitive strategies and motivation towards reading, an improvement in the level of reading comprehension of the participants was obtained.

Keywords: Reading comprehension; cognition; achievement motivation; intervention

\section{Introducción}

La comprensión lectora es un tema que ha recibido mucha atención de parte de la comunidad educativa por la relevancia que adquiere en el proceso educativo y en el desempeño de los estudiantes. En este caso, el Instituto de Estadística de la UNESCO menciona que más de la mitad de los niños y adolescentes del mundo, unos 617 millones, no obtienen las competencias básicas en lectura y matemáticas (UNESCO, 2017); es decir, que seis de cada 10 no están aprendiendo. Mientras que en África Subsahariana el 88\% de los niños y adolescentes no alcanzan las competencias lectoras, en América Latina y el Caribe ese valor es del 36\%, en tanto que en América del Norte y Europa occidental la cifra desciende a un $14 \%$.

La lectura es un medio para acceder a la información y una vía para generar conocimiento, un individuo que gusta de la lectura y posee habilidades lectoras está capacitado para desarrollarse en la sociedad actual (Osorio et al., 2018). Vivimos en una época denominada la sociedad del conocimiento, por ende, es de vital importancia el desarrollo de la lectura como una competencia que permita la participación de los ciudadanos en las actividades sociales y culturales (De Souza, 2016). Sin embargo, los resultados de las evaluaciones internacionales dan evidencia de la asimetría que existe entre los países desarrollados y los que están en vías de desarrollo.

De acuerdo con los datos de la prueba PISA 2018, la media de lectura obtenida por los países de la OCDE fue de 487 puntos (OECD, 2019). De los países asiáticos, China obtuvo el mejor puntaje a nivel mundial, con una media de comprensión lectora de 555 
puntos. Entre los países latinoamericanos y del Caribe que participaron, Chile es el mejor posicionado con una media de 452 puntos. República Dominicana, con 342 puntos, ocupa el último lugar. México se ubica en una posición intermedia, con 420 puntos.

La situación adquiere un nivel preocupante cuando comparamos a los países Latinoamericanos con Estados Unidos $(M=505)$ o con Canadá $(M=520)$, al considerar que 30 puntos de diferencia equivalen a un año académico de estudios. La situación se hace todavía más alarmante cuando se observa una disminución en los resultados de comprensión lectora de México en la prueba PISA desde el 2009 (Backhoff, 2019).

\section{Comprensión lectora}

En las últimas décadas se ha avanzado mucho en cuanto al conocimiento de los procesos de lectura, especialmente del rol del lector y de las estrategias que utiliza. Esta concepción ha evolucionado desde un enfoque que priorizaba el proceso lector y las dificultades asociadas, a uno centrado en la comprensión, en el cual leer implica necesariamente comprender. Así, según Mengual (2017), comprender significa extraer el significado y hacer que sea "consciente en nuestra mente, llegando a interactuar con el autor del texto” (p. 19). Este proceso, al relacionarse con los conocimientos previos del lector, brindan “coherencia al texto para facilitar su comprensión e interpretación” (p. 19).

La comprensión lectora, es una habilidad que permite asimilar la esencia del texto, formular conclusiones, realizar inferencias y usar esa información para el logro de objetivos académicos o personales (Cano, 2015). Un lector competente es capaz de razonar, interpretar el contenido y comprender los distintos niveles de un texto, aunado a lo anterior, despliega una actitud activa y participativa en sus procesos de lectura (Alaís et al., 2015).

En la lectura, suceden diversos procesos cognitivos en la mente del lector para lograr la comprensión lectora. Comprender un texto es uno de los aspectos básicos que todo alumno debería desarrollar, pues lo capacita para que en el futuro logre otros procesos de aprendizaje cognitivos más complejos como el razonamiento y la lógica (Neira et al., 2015).

Dado que estamos en la sociedad del conocimiento, en el que la información cae prontamente en caducidad (Núñez, 2016), es necesaria una educación basada en competencias, con un énfasis que incluye la competencia lingüística. Esta es una habilidad básica en los estudiantes, ya que por su carácter transversal impacta de forma directa sobre su aprendizaje. Comprender un texto capacita al alumno para el logro de aprendizajes 
cognitivos más complejos como el razonamiento y la lógica, y, además, sirve como base para acceder a otras competencias (Neira et al., 2015).

\section{Motivación}

Una idea muy desarrollada en la actualidad plantea que un individuo motivado será más productivo que otro que no lo está. Por ende, aumentar la motivación de los estudiantes hacia el aprendizaje se ha convertido en una preocupación constante de los docentes de educación básica y superior (Orbegoso, 2016).

De acuerdo con Huilcapi et al. (2017), el constructo motivación tiene su origen en el latín motus, que significa movido. La motivación se define como "el señalamiento o énfasis que se descubre en una persona hacia un determinado medio de satisfacer una necesidad, creando o aumentando con ello el impulso necesario para que ponga en obra ese medio o esa acción” (p. 316).

Según Alemán et al. (2018), la motivación es un factor clave en el proceso de aprendizaje. Un alumno motivado es capaz de plantearse objetivos definidos y delimitar las estrategias para alcanzarlos. Es emprendedor, innovador y conocedor de sus áreas de debilidad y fortaleza. Enfrenta las dificultades con seguridad, busca soluciones alternas a los problemas que encara y es una persona proactiva. Además, constituye un factor que explica el inicio, dirección y persistencia hacia una determinada meta académica (Alemany et al., 2015).

Un factor que se relaciona fuertemente con la comprensión lectora y el uso de estrategias es la motivación hacia la lectura, esta es una variable de primera importancia en un contexto donde la gran parte de los conocimientos académicos están expresados de forma escrita (Muñoz, 2016).

La motivación intrínseca es una cualidad de los individuos que leen por placer, en tanto la motivación extrínseca caracteriza a los que leen solo por cumplir requisitos académicos (Guerra y Guevara, 2017). Por ello, los estudiantes motivados leen con mayor frecuencia y esa lectura frecuente facilita el desarrollo de competencias lectoras superiores, estos lectores normalmente desarrollan un alto sentido de autoeficacia algo que no sucede con aquellos lectores que al tener menores habilidades para leer se desmotivan rápidamente y por lo tanto dejan de leer. Esta conducta genera un círculo vicioso que les impide tener 
oportunidades de mejorar sus habilidades lectoras, siendo una de las causas principales del bajo nivel de desempeño lector (Navarro et al., 2018).

\section{Estrategias metacognitivas}

En los últimos años aumentaron las investigaciones sobre las estrategias metacognitivas y su aplicación se extendió a distintas especialidades académicas como un aliado en el proceso enseñanza-aprendizaje.

Para Larrañaga y Yubero (2015), “Uno de los temas que más preocupan en la actualidad es la comprensión lectora. La conceptualización del proceso de comprensión se ha enriquecido en los últimos años, incluyendo los aspectos metacognitivos como herramienta esencial para la optimización de su desarrollo” (p. 23).

De acuerdo con López (2015), la metacognición incluye estrategias que supervisan los procesos que realiza la cognición. El sujeto debe conocer y autorregular sus procesos mentales con la finalidad de programar de manera consciente estrategias para el aprendizaje, resolución de problemas y toma de decisiones.

En procesos de lectura, Al-Mekhlafi (2018) menciona que en muchos estudios el término estrategia hace alusión a operaciones mentales realizadas por los lectores para obtener sentido a la lectura, las cuales actúan como recursos para la resolución de problemas al afrontar la lectura de un texto.

Las estrategias metacognitivas hacia la lectura se definen como el acompañamiento consciente del lector a su proceso de lectura, lo cual le permite detectar deficiencias, realizar intervenciones cuando se requieren y hacer una evaluación del proceso utilizado para la compresión del texto (Aşıkcan y Saban, 2018).

De acuerdo con Muñoz y Ocaña (2017), las estrategias metacognitivas enfocadas a la lectura permiten que el alumno pueda tener control sobre el texto, definir objetivos, establecer las mejores estrategias, supervisar el proceso lector y evaluar los resultados. Por ello, los estudiantes requieren utilizar estrategias metacognitivas para comprender textos difíciles, debido a que estas estrategias incluyen selección, ejecución y control de estrategias cognitivas. Además, su uso constante permite desarrollar la competencia lectora (Filiz et al., 2018).

En este sentido, las estrategias metacognitivas hacia la lectura pueden catalogarse según el momento de uso, de la siguiente manera: (a) antes de la lectura, se plantean los 
propósitos y las estrategias que se usarán para lograrlos; (b) durante la lectura o supervisión, en esta fase las estrategias están orientadas a controlar el proceso lector a través de actividades como subrayar, tomar apuntes, releer en caso de ser necesario, entre otras; (c) después de la lectura, esta etapa se centra en la evaluación, se pretende verificar si los resultados corresponden con los objetivos iniciales, se realizan estrategias como elaborar resúmenes, organizadores gráficos o cuestionarios (Khoshsima y Amiri, 2015; Ramírez et al., 2015 ).

\section{Relación entre las variables de estudio}

Según Sedano (2015), la comprensión lectora está ligada a la motivación hacia la lectura; una variable separada de la otra resulta insuficiente. No sirve de nada estar con deseos de leer si al comenzar la lectura los párrafos resultan incomprensibles; con un patrón como este, al final la falta de comprensión terminará matando la motivación.

En un estudio de Guerra et al. (2014), los autores señalan que además de la relación que existe entre las estrategias metacognitivas y la comprensión lectora, estas tienen una relación con ciertos aspectos de la motivación, de forma específica con la motivación intrínseca y extrínseca. Los autores mencionan que quienes tienen una meta motivacional intrínseca utilizan mejores estrategias de lectura y logran mejores niveles de comprensión lectora.

Por su parte, Espino y Sánchez (2017) estudiaron si la motivación y las emociones personales tenían relación con la comprensión lectora. Se encontró una correlación positiva de ambas variables con la comprensión lectora, pero solo fue significativa con la variable motivación. Por lo tanto, se concluyó que la motivación se relaciona positivamente en los niveles de comprensión lectora de los estudiantes.

Castrillón et al. (2020) aplicaron un programa de intervención basado en la enseñanza de estrategias metacognitivas en estudiantes de décimo grado. Su propósito fue comprobar si la intervención producía mejoras en la comprensión lectora. El instrumento aplicado media tres dimensiones de comprensión lectora: literal, inferencial y crítico. Los resultados revelaron un incremento en los tres niveles de comprensión, pero ese aumento sólo fue significativo para el nivel literal y crítico. Estos resultados dan evidencia que el uso de estrategias metacognitivas hacia la lectura mejora los niveles de comprensión lectora. 


\section{Metodología}

\section{Diseño}

Este trabajo corresponde a un enfoque cuantitativo, en el que se realizó una recolección de datos para probar la hipótesis de estudio y su posterior análisis mediante el uso de la estadística para determinar el comportamiento de los participantes (Sánchez et al., 2018). Fue un estudio cuasi experimental, antes (pretest) y después (postest) con un solo grupo, los sujetos no se seleccionaron al azar, sino que dicho grupo ya estaba formado antes de la intervención (Echevarría, 2016).

\section{Participantes}

En esta investigación, la población estuvo compuesta por 18 estudiantes de una escuela preparatoria abierta nocturna en el Estado de Nuevo León, México. La muestra fue de tipo censal, pues se esperó que todos los estudiantes matriculados participaran del estudio. Sin embargo, solo aquellos que contestaron voluntariamente el cuestionario fueron tomados en cuenta a los fines de esta investigación. Como criterios de eliminación, se excluyeron a los alumnos que no presentaron el pretest o el postest y a los estudiantes que dejaron de contestar más del 20\% de los instrumentos de medición aplicados.

\section{Instrumentos}

La comprensión lectora se valoró mediante el Test de Cloze, que consiste en proporcionar a los alumnos un texto completo de entre 250 a 300 palabras. Posteriormente, se retira el texto y se les proporciona el mismo texto, pero con la omisión de ciertas palabras. Los alumnos deben completarlo con las palabras que recuerden; pueden usarse las palabras exactas del texto o sinónimos, según el criterio del evaluador. Es necesario dejar intactas la primera y la última oración del texto. A partir de la segunda oración se suprime cada quinta palabra hasta llegar a la penúltima oración. Este tipo de omisión sistemática permite que las palabras omitidas sean hechas al azar, a fin de mantener el nivel de confiabilidad y evitar que el examinador imponga su criterio al elegir las palabras. En el texto se omitieron 38 palabras que los estudiantes debían completar.

Acerca de la confiabilidad y la validez, el Test de Cloze ha sido examinado por diferentes investigadores utilizando diversas técnicas. Con base en la fórmula KuderRichardson se han obtenido coeficientes de confiabilidad de 0.90 (Calderón y Quijano, 2010). 
En otro estudio, realizado con estudiantes universitarios mexicanos, Ruvalcabar y Rodríguez (2019) obtuvieron un coeficiente alfa de Cronbach de .93.

Para la medición de la motivación, se utilizó la Escala Atribucional de Motivación de Logro General (EAML-G). La prueba fue adaptada y validada por Durán y Pujol (2013) en una población de estudiantes universitarios venezolanos, quienes obtuvieron una medida de consistencia interna medida con el alfa de Cronbach igual a .82. Otro estudio, llevado a cabo por Ruvalcabar y Rodríguez (2019) con estudiantes universitarios mexicanos, reveló un coeficiente alfa de Cronbach de .88 .

El EAML-G consta de 18 declaraciones valoradas con una escala Likert de seis puntos, donde 1 representa una valoración baja o negativa y 6 una valoración alta o positiva. La escala está dividida en cuatro dimensiones: (a) características de la tarea, (b) interés/esfuerzo, (c) capacidad y (d) evaluación del docente.

Las estrategias metacognitivas se valoraron con el IEMG. La versión traducida al español y validada por Huertas et al. (2014) con estudiantes de grados décimo y undécimo de instituciones educativas en la ciudad de Bogotá, obtuvo un coeficiente alfa de Cronbach de .94. Por su parte, Ruvalcabar y Rodríguez (2019), en un estudio realizado con estudiantes universitarios mexicanos, obtuvieron un coeficiente alfa de Cronbach de .88 .

El instrumento consta de 33 ítems, valorados mediante una escala Likert de cuatro puntos, con ocho dimensiones: (a) conocimiento declarativo metacognitivo, (b) conocimiento procedimental metacognitivo, (c) conocimiento condicional metacognitivo, (d) planeamiento, (e) manejo de la información, (f) control de errores, (g) monitoreo de la comprensión y (h) autoevaluación posterior. En esta escala, una valoración de 1 significa no lo hago nunca y 4 significa lo hago siempre. Una mayor valoración implica, respectivamente, una mejor situación en cada una de las escalas. El proceso de recolección de datos se efectuó en el mes de febrero del año 2020.

\section{Procedimiento}

Previo a la intervención, los estudiantes contestaron el Test de Cloze, la Escala Atribucional de Motivación de Logro General (EAML-G) y el Inventario de Estrategias Metacognitivas Generales (IEMG). Luego, los estudiantes participaron de una capacitación basada en motivación y estrategias metacognitivas para mejorar la comprensión lectora. 
Dicha intervención consistió en sesiones de 45 minutos, durante 14 semanas, que incluyeron sesiones teóricas y ejercicios prácticos de manera alternada. Por último, se realizó una nueva administración de los instrumentos, a fin de verificar si existía una diferencia significativa en la comprensión lectora entre la primera y la segunda medición, como consecuencia de la intervención.

\section{Intervención}

Respecto a la intervención, esta se fundamentó en las ideas de Schunk (2012), el menciona que la metacognición incluye dos conjuntos de habilidades que están muy relacionadas. En primer lugar, se debe entender qué habilidades, estrategias y recursos requiere cada tarea. En este grupo se incluye la localización de las ideas principales, el repaso de la información, la formación de asociaciones o imágenes, el uso de técnicas de memoria, la organización del material, la toma de notas y el subrayado. En segundo lugar, se debe saber cómo y cuándo utilizar esas habilidades y estrategias para asegurarse de tener éxito en el cumplimiento de la tarea. Algunas de esas actividades de monitoreo consisten en verificar el nivel de comprensión, pronosticar los resultados, evaluar la eficacia de los esfuerzos, decidir cómo administrar el tiempo y revisar o cambiar a otras actividades para superar las dificultades. La metacognición se activa cuando los estudiantes establecen metas, evalúan su progreso hacia ellas y hacen las correcciones necesarias. Todas estas ideas se aplicaron al área de la comprensión lectora.

Respecto a la motivación, Schunk (2012) menciona que existe una relación directa entre la motivación y el aprendizaje y que se influyen mutuamente. La motivación de los alumnos puede influir en lo que aprenden y en la forma cómo lo aprenden. A su vez, a medida que los estudiantes aprenden y perciben que se vuelven cada vez más hábiles, se sienten motivados para seguir aprendiendo. Estos postulados dieron origen a la elaboración y a la selección de los temas, los cuales se describen en la tabla 1. 


\section{Tabla 1}

Descripción de los módulos de la intervención

\begin{tabular}{|c|c|c|}
\hline Nombre de la sesión & Objetivo & Actividades \\
\hline Introducción de la intervención & $\begin{array}{l}\text { Conocer las motivaciones de los } \\
\text { compañeros que conforman el } \\
\text { grupo. }\end{array}$ & $\begin{array}{l}\text { Dinámica de conocimiento } \\
\text { “sondeo de motivaciones”. }\end{array}$ \\
\hline Autoconocimiento & $\begin{array}{l}\text { El alumno aprenda a reconocer sus } \\
\text { fortalezas y debilidades al iniciar } \\
\text { una actividad de aprendizaje. }\end{array}$ & $\begin{array}{l}\text { Lista de actividades mentales que } \\
\text { incluya las áreas que domina y } \\
\text { las que tiene que desarrollar. }\end{array}$ \\
\hline Nivel de velocidad lectora & $\begin{array}{l}\text { Conocer el nivel de velocidad } \\
\text { lectora de los alumnos. }\end{array}$ & $\begin{array}{l}\text { Brindar estrategias que permitan } \\
\text { mejorar los niveles de velocidad } \\
\text { lectora e influir de forma positiva } \\
\text { en sus niveles de estudio. }\end{array}$ \\
\hline Estrategias de lectura & $\begin{array}{l}\text { Reconocer e identificar el objetivo } \\
\text { al emprender una lectura de } \\
\text { estudio. }\end{array}$ & $\begin{array}{l}\text { Enseñar estrategias } \\
\text { metacognitivas diseñadas para } \\
\text { aplicarse antes, durante y después } \\
\text { de un periodo de lectura. }\end{array}$ \\
\hline $\begin{array}{l}\text { Elaboración de organizadores } \\
\text { gráficos }\end{array}$ & $\begin{array}{l}\text { El alumno obtenga la competencia } \\
\text { de elaborar organizadores gráficos } \\
\text { como forma de organizar } \\
\text { información. }\end{array}$ & $\begin{array}{l}\text { Elaborar un organizador gráfico a } \\
\text { partir de una lectura corta. }\end{array}$ \\
\hline Monitoreo & $\begin{array}{l}\text { El alumno desarrolle la habilidad } \\
\text { de ser consciente de su proceso de } \\
\text { aprendizaje. }\end{array}$ & $\begin{array}{l}\text { Enseñanza de estrategias } \\
\text { metacognitivas orientadas al } \\
\text { monitoreo de los procesos } \\
\text { implicados en el aprendizaje. }\end{array}$ \\
\hline Autoevaluación & $\begin{array}{l}\text { El alumno realice críticas a su } \\
\text { proceso de aprendizaje y elabore } \\
\text { metareflexiones del mismo. }\end{array}$ & $\begin{array}{l}\text { Durante la semana realizar un } \\
\text { diario o bitácora de aprendizaje } \\
\text { de las materias vistas en el aula. }\end{array}$ \\
\hline
\end{tabular}

\section{Análisis estadístico}

Para efectuar el análisis estadístico de los datos, se utilizó el software SPSS versión 22. Los datos fueron sistematizados mediante tablas descriptivas que incluyen la media y la desviación estándar de los ítems con mayor valoración. Para los resultados inferenciales se utilizó la prueba t de Student.

\section{Consideraciones éticas}

El estudio contó con la aprobación previa del director de la institución. Los estudiantes fueron invitados a través de sus docentes y el investigador hizo una descripción de los objetivos y actividades para motivar su participación. Luego, se procedió a la administración de los instrumentos en el momento y lugar acordado, y se verificó que los estudiantes dispusieran de al menos 45 minutos de tiempo. Se explicó que la actividad no era un examen, por lo cual no había respuestas verdaderas o falsas, y que la participación sincera y a 
conciencia, permitiría tomar mejores decisiones al investigador. Se informó que la información obtenida sería utilizada de manera confidencial, y que la participación era voluntaria. Cabe mencionar que este estudio contó con la aprobación del Comité de Ética de la Dirección de Posgrado e Investigación de la Universidad de Montemorelos.

\section{Resultados}

Con relación a las características sociodemográficas de los participantes, estos fueron 18 estudiantes, cuyas edades estaban comprendidas entre los 16 y 60 años de edad $(M=$ 21.88, $D E=11.35)$, se observó que 7 sujetos fueron de género masculino (38.9\%) y 11 fueron de género femenino (61.1\%). En cuanto al estado civil, 14 estudiantes eran solteros $(n=14$, 77.8\%) y 4 eran casados (22.2\%). La distancia en el rango de edad puede entenderse mejor al considerar que los estudiantes cursan la preparatoria abierta en turno nocturno, pues la mayoría de ellos trabajan durante el día.

La Tabla 2 presenta los datos descriptivos de los ítems de las variables motivación y estrategias metacognitivas con mayor valoración. Se puede observar que, tanto en el pretest como en el postest, las declaraciones mejor valoradas de la variable motivación se referían a cuánto interés tienen por estudiar este semestre, cuán importantes eran las buenas notas obtenidas en este semestre, cuanta motivación tienen los alumnos por obtener buenas notas y cuántas ganas tienen de aprender este semestre. En las estrategias metacognitivas, se resalta que los alumnos estudian más lento cuando el tema es difícil, prestan atención conscientemente a las explicaciones de temas importantes, confían en sus capacidades y pueden motivarse a aprender cuando lo necesitan aunque se preguntan si han alcanzado sus objetivos una vez que han terminado un examen. 
Tabla 2

Variables observadas con mayor valoración

\begin{tabular}{lrrrr}
\hline \multicolumn{1}{c}{ Variable } & \multicolumn{2}{c}{ Pretest } & \multicolumn{2}{c}{ Postest } \\
\cline { 2 - 5 } & $M$ & $D E$ & $M$ & $D E$ \\
\hline Motivación & & & & \\
8. ¿Cuán importantes son para ti las buenas notas en este semestre? & 5.61 & 0.78 & 5.67 & 0.97 \\
9. ¿Cuánto interés tienes por estudiar este semestre? & 5.72 & 0.46 & 5.50 & 0.86 \\
12. ¿Cuánto afán tienes de sacar buenas notas? & 5.61 & 0.61 & 5.72 & 0.67 \\
16. ¿Cuántas ganas tienes de aprender este semestre? & 5.44 & 1.42 & 5.56 & 0.98 \\
Estrategias metacognitivas & & & & \\
6. Me pregunto si conseguí alcanzar mis objetivos una vez que he terminado. & 3.06 & 1.43 & 3.28 & 0.57 \\
8. Estudio más lento cuando el tema es difícil. & 2.67 & 1.53 & 3.67 & 0.77 \\
25. Confío en mis capacidades. & 2.67 & 1.24 & 3.44 & 0.62 \\
30. Presto atención conscientemente a las explicaciones de conceptos & & & & \\
importantes. & 2.89 & 1.28 & 3.50 & 0.62 \\
31. Puedo motivarme a aprender cuando lo necesito. & 3.00 & 1.33 & 3.44 & 0.86 \\
\hline
\end{tabular}

Se validó el supuesto de distribución normal de la diferencia de las puntuaciones del postest y el pretest de la variable comprensión lectora con el Test de Shapiro-Wilk (SW = .952, $p=.461)$. Al comparar el nivel de comprensión lectora entre el pretest $(M=8.28, D E$ =6.85) y el postest $(M=20.06, D E=9,70)$, se observó que existe una diferencia significativa entre ambas mediciones con un tamaño del efecto grande (Tabla 3). Además, la potencia estadística de la prueba fue de .9680 .

Acerca de la variable autopercepción de las estrategias metacognitivas, se validó el supuesto de distribución normal de la diferencia de las puntuaciones del pretest y el postest. Se encontró evidencia de que se viola el supuesto de distribución normal $(S W=.879, p=$ .025), por lo que se utilizó la prueba no paramétrica de Wilcoxon con un tamaño del efecto medio (Tabla 3) y una potencia estadística igual a .633.

También se validó el supuesto de distribución normal de la diferencia de las puntuaciones del postest y el pretest de la variable autopercepción de la motivación. Se observó que se cumple el supuesto de distribución normal ( $S W=.974, p=.868)$, aunque no se encontró diferencia significativa entre el pretest y el postest, con un tamaño del efecto pequeño (Tabla 3). La Tabla 3 presenta los datos descriptivos de las variables observadas juntos con los resultados de las pruebas de hipótesis. 
Tabla 3

Comparación de las variables observadas en el pretest y postest

\begin{tabular}{lcccccc}
\hline \multicolumn{1}{c}{ Variables } & \multicolumn{1}{c}{ Prueba de hipótesis } & $M$ & $D E$ & $M d n$ & $\alpha$ & Normalidad \\
\hline Comp. lectora_Pretest & & 8.28 & 6.85 & 6.500 & 0.903 & \\
Comp_lectora_Postest & $t_{(17)}=3.653, p=.002, d=0.86$ & 20.06 & 9.70 & 19.000 & 0.946 & $0.461^{\text {a }}$ \\
Motivación Pretest & & 4.82 & 0.72 & 4.889 & 0.881 & \\
Motivación Postest & $t_{(17)}=-0.341, p=.737, d=0.008$ & 4.90 & 0.60 & 5.111 & 0.770 & $0.025^{\text {b }}$ \\
Est. Metacog. Pretest & & 2.40 & 0.96 & 2.682 & 0.979 & \\
Est. Metacog. Postest & $W=30.00, p=.017, r=0.649 *$ & 2.97 & 0.40 & 3.015 & 0.902 & $0.868^{\text {c }}$ \\
\hline
\end{tabular}

Nota. La normalidad corresponde a las diferencias entre el Postest y el Pretest de cada una de las variables. ${ }^{\text {a }}$ Comprensión lectora, ${ }^{\mathrm{b}}$ Motivación, ${ }^{\mathrm{c}}$ Estrategias metacognitivas. $* \mathrm{r}=$ Correlación biserial

\section{Discusión}

El objetivo de esta investigación fue conocer si existía diferencia significativa entre el pretest y el postest de comprensión lectora después de realizar una intervención basada en la motivación y las estrategias metacognitivas hacia la lectura. En los resultados obtenidos, se encontró que existe diferencia significativa en los niveles de comprensión lectora, después de haber llevado a cabo una intervención basada en el uso de estrategias metacognitivas y motivación. Este hallazgo concuerda con Hernández (2015), quien indica que el empleo de estrategias metacognitivas mejora la comprensión lectora, ya que se correlacionan significativamente (Gómez y Sanabria, 2018; Alcas, 2019). Asimismo, cuando los estudiantes utilizan las estrategias metacognitivas relacionadas con la comprensión de textos, adquieren de forma progresiva una mayor comprensión lectora (Nasab y Motlagh, 2015).

Contrario a los resultados anteriores, Paba y González (2014), encontraron, a partir de un estudio con alumnos de décimo grado, que no existe relación entre la actividad metacognitiva y la comprensión lectora, ni de manera global ni por componentes; esto indica que el nivel de comprensión lectora obtenido por los alumnos no se relaciona con el conocimiento o el uso de algunas estrategias metacognitivas. Resultados afines fueron hallados por Meniado (2016), quien señala que no hay relación alguna entre los procesos metacognitivos y la comprensión lectora, pero sí una relación positiva entre las estrategias metacognitivas y la motivación por la lectura.

En lo referente a la motivación, no se encontró diferencia significativa en la media de esta variable, ya que los resultados mantuvieron valores similares en el pretest y el postest. 
Es importante mencionar que en el pretest se obtuvieron valores relativamente altos de motivación, lo que puede indicar que los alumnos enfrentan la actividad académica con una motivación alta y, por tal motivo, no hubo cambios significativos. Además, al correlacionar la motivación con la comprensión lectora, no se halló relación significativa entre el pretest y el postest, lo cual difiere de los hallazgos de Espino y Sánchez (2017).

Respecto a los resultados obtenidos en el manejo de la información metacognitiva, estos coinciden con los obtenidos por Mato et al. (2017), quienes mencionan que las estrategias metacognitivas permiten examinar y conectar los conocimientos previos con información nueva. Munayco (2018) encontró que los organizadores gráficos tienen una gran contribución en la mejoría de la comprensión lectora, ya que permiten organizar la información de mejor forma y aprovechar mejor el contenido. El conocimiento procedimental metacognitivo está estrechamente ligado a la regulación de los procesos cognitivos que suceden durante las fases de planificación, control y evaluación, es decir, el conocimiento de la forma apropiada de resolver un problema o de mejorar el proceso de aprendizaje (Valenzuela, 2019).

En relación con el planteamiento metacognitivo, los lectores maduros ejecutan ciertas estrategias antes de la lectura. Estas estrategias consisten en establecer objetivos y utilizar estrategias para alcanzar la meta de aprendizaje. El sujeto controla su proceso de aprendizaje formulando objetivos claros: qué se pretende lograr, con qué recursos humanos o materiales se cuenta, emplear estrategias de pre-lectura como la exploración textual (revisar las partes claves del texto) o el ojeado (lectura rápida del texto), activar los conocimientos previos y cómo resolver la relación tiempo-esfuerzo que la tarea demanda (Channa et al., 2015).

Los resultados obtenidos muestran coherencia con los de Cabero et al. (2018), quienes encontraron diferencias significativas en los niveles de estrategias metacognitivas de lectura entre el pretest y el postest de un grupo control y otro experimental, después de una intervención basada en material educativo con soporte tecnológico. Las estrategias metacognitivas relacionadas con la lectura son un apoyo importante para los estudiantes con sobreedad que cursan la escuela preparatoria nocturna. La intervención logró mejorar sus estrategias, lo que se vio reflejado en mejoras significativas de la comprensión lectora.

Con estos datos, los resultados del estudio aportan información relevante y dan cuenta de la necesidad de realizar un apoyo constante en estrategias que mejoren la comprensión 
lectora, especialmente en estudiantes que trabajan y hacen un esfuerzo para continuar sus estudios durante las horas de la noche.

Los alumnos que asisten a escuelas nocturnas normalmente combinan sus estudios con muchas horas previas de trabajo, por lo tanto, ellos requieren tener desarrolladas estas estrategias que les permitan aprovechar al máximo los pequeños lapsos de tiempo que dedican al estudio de sus materias. El desarrollo de estas estrategias y su implementación permitirán que los estudiantes puedan comprender de mejor forma sus lecturas, mejorar su rendimiento académico y continuar con sus estudios de forma más provechosa.

\section{Conclusiones}

Considerando los resultados obtenidos del estudio se concluye que los alumnos de una escuela preparatoria abierta nocturna del estado de Nuevo León (México) obtuvieron una mejora significativa en su nivel de comprensión lectora después de participar de una intervención basada en la motivación y las estrategias metacognitivas.

Aun cuando la población participante en el estudio fue pequeña, se cumplió el supuesto de distribución normal de la diferencia de las puntuaciones del pretest y el postest de la variable comprensión lectora. Además, la potencia estadística de la prueba fue muy importante. Una potencia es adecuada para una prueba estadística cuando es superior a 0,80 (80\%). Es decir, existe un 20\% de probabilidad de aceptar la hipótesis nula cuando esta es en realidad falsa $(\beta=.20)$. Se estima que un valor mas bajo implicaría un riesgo muy grande de incurrir en un error Tipo II. Así, la potencia estadística constituye un índice de la validez de los resultados estadísticos (Cohen, 1988; Cohen, 1992).

Para futuros estudios se recomienda, replicar la investigación con poblaciones más amplias y de distintos centros educativos, tener un grupo control que permita comparar resultados de manera confiable con el grupo experimental y tomar en cuenta ciertas variables sociodemográficas y académicas para tener una visión más amplia y analizar cómo estas variables se relacionan con el tema de estudio.

\section{Referencias}

Alaís Grillo, A., Leguizamón Sotto, D. V. y Sarmiento Ceballos, J. I. (2015). Comprensión lectora y desarrollo de estrategias cognitivas con el apoyo de un recurso TIC. Revista Educación y Desarrollo Social, 9(1), 132-143. https://doi.org/10.18359/reds.556 
Alcas Zapata, N., Alarcón Diaz, M. A., Alarcón Diaz, H. H., Gonzáles LLontop, R. y Rodríguez Fuentes, A. (2019). Estrategias metacognitivas y comprensión lectora en estudiantes universitarios. Apuntes Universitarios, 9(1), 36-45. https://doi.org/10.17162/au.v9i1.348

Alemán Marichal, B., Navarro de Armas, O. L., Suárez Díaz, R. M., Izquierdo Barceló, Y. y Encinas Alemán, T. C. (2018). La motivación en el contexto del proceso enseñanzaaprendizaje en carreras de las ciencias médicas. Revista Médica Electrónica, 40(4), 1257-1270.

http://www.revmedicaelectronica.sld.cu/index.php/rme/article/view/2307

Alemany Arrebola, I., Campoy Barreiro, C., Ortiz Gómez M. M. y Benzaquén Chocrón, R. (2015). Las orientaciones de meta en el alumnado de secundaria: Un análisis en un contexto multicultural. Publicaciones, 45, 83-100. https://revistaseug.ugr.es/index.php/publicaciones/article/view/5790

Al-Mekhlafi, A. M. (2018). EFL Learners metacognitive awareness of reading strategies. International Journal of Instruction, 11(2), 297-308. https://doi.org/10.12973/iji.2018.11220a

Aşıkcan, M. y Saban, A. (2018). Prospective teacher's metacognitive awareness levels of reading strategies. Cypriot Journal of Educational Science, 8(1), 23-30. https://doi.org/10.18844/cjes.v13i1.3310

Backhoff Escudero, E. (2019, diciembre 9). México en PISA 2018. Nexos. https://educacion.nexos.com.mx/?p=2059

Cabero Almenara, J., Piñero Virué, R. y Reyes Rebollo, M. M. (2018). Material educativo multimedia para el aumento de estrategias metacognitivas de comprensión lectora. $\begin{array}{lll}\text { Perfiles } \quad \text { Educativos, } & \text { 40(159), } 159 .\end{array}$ http://www.perfileseducativos.unam.mx/iisue_pe/index.php/perfiles/article/view/58 $042 / 51635$

Calderón Ibañez, A. y Quijano Peñuela, J. (2010). Características de comprensión lectora en estudiantes universitarios. Revista Estudios Socio-Jurídicos, 12(1), 337-364. https://revistas.urosario.edu.co/index.php/sociojuridicos/article/view/1195

Cano Vela, A. G. (2015). Estrategias de comprensión lectora para estudiantes de magisterio. Educatio Siglo XXI, 33(2), 123-140. https://doi.org/10.6018/j/232721

Castrillón Rivera, E. M., Morillo Puente, S. y Restrepo Calderón, L. A. (2020). Diseño y aplicación de estrategias metacognitivas para mejorar la comprensión lectora en estudiantes de secundaria. Ciencias Sociales y Educación, 9(17), 203-231. https://doi.org/10.22395/csye.v9n17a10

Channa, M. A., Nordin, Z. S., Siming, I. A., Chandio, A. A. y Koondher, M. A. (2015). Developing reading comprehension through metacognitive strategies: A review of 
previous studies. English Language Teaching, 8(8), 181-186. http://doi.org/10.5539/elt.v8n8p181

Cohen, J. (1988). Statistical Power Analysis for the Behavioral Sciences. (2da ed.). New Jersey: Lawrence Erlbaum Associates.

Cohen, J. (1992). Cosas que he aprendido (hasta ahora). Anales de Psicología, 8(1-2), 3-18. https://revistas.um.es/analesps/article/view/28521

De Souza Siqueira, T. G. (2016). Lectura, biblioteca e inclusión social: Importancia de la promoción de la lectura en comunidades ribereñas en Amazonas, Brasil. Información, Cultura y Sociedad, 34, 93-106. https://doi.org/10.34096/ics.i34.2256

Durán-Aponte, E. y Pujol, L. (2013). Escala atribucional de motivación de logro general (EAML-G): adaptación y análisis de sus propiedades psicométricas. Estudios Pedagógicos (Valdivia), 39(1), 83-97. https://doi.org/10.4067/s071807052013000100005

Echevarría, H. D. (2016). Diseños de investigación cuantitativa en psicología y educación (1ra ed.). UniRio. https://www.unrc.edu.ar/unrc/comunicacion/editorial/repositorio/ 978-987-688-166-1.pdf

Espino Dragustinovis, D. y Sánchez Tovar, Y. (2018). La motivación y las emociones, factores clave para la comprensión lectora universitaria. Educiencia, 2(3), 22-26. https://educiencia.uat.edu.mx/index.php/Educiencia/article/view/34

Filiz, S. B., Erol, M. y Erol, A. (2018). Investigating the correlation between the frequency of using metacognitive reading strategies and non-routine problem solving successes in fifth grade students. Universal Journal of Educational Research, 6(8), 1795-1802. https://doi.org/10.13189/ujer.2018.060824

Gómez, J. H. y Sanabria Marín, K. M. (2018). Estrategias Metacognitivas: Engranaje de la comprensión lectora. La Tercera Orilla, (21), 51-64. https://doi.org/10.29375/21457190.3475

Guerra García, J. y Guevara Benítez, C. Y. (2017). Variables académicas, comprensión lectora, estrategias y motivación en estudiantes universitarios. Revista Electrónica de Investigación $\quad$ Educativa, $19(2), \quad 78-90$. https://doi.org/10.24320/redie.2017.19.2.1125

Guerra García, J., Guevara Benítez, C. y Robles Montijo, S. (2014). Validación del inventario de estrategias metacognoscitivas y motivación por la lectura (IEMML) en estudiantes de psicología. Psicogente, 17(31), 17-32. https://doi.org/10.17081/psico.17.31.419

Hernández Vásquez, N. E. (2015). Estrategias metacognitivas y comprensión de lectura en los estudiantes de Psicología de una Universidad Nacional (Tesis de maestría). Universidad Nacional Federico Villareal, San Miguel, Perú. 
Huertas Bustos, A. P., Vesga Bravo, G. J. y Galindo León, M. (2014). Validación del instrumento inventario de habilidades metacognitivas (MAI) con estudiantes colombianos. Praxis \& Saber, 5(10), 56-74. https://doi.org/10.19053/22160159.3022

Huilcapi Masacon, M. R., Castro López, G. A. y Jácome Lara, G. A. (2017). Motivación: las teorías y su relación en el ámbito empresarial. Dominio de las Ciencias, 3(2), 311333. https://doi.org/10.23857/dc.v3i2.332

Khoshsima, H. y Amiri Samani, E. (2015). The most frequent metacognitive strategies used in Reading comprehension among ESP learners. Advances in Languaje and Literary Studies, 6(1), 180-188. https://doi.org/10.7575/aiac.alls.v.6n.1p.180

Larrañaga, E. y Yubero S. (2015). Evaluación de las estrategias metacognitivas de comprensión de textos narrativos. Ocnos, 14, 18-27. https://doi.org/10.18239/ocnos_2015.14.02

López Jurado, A. L. (2015). Estrategias metacognitivas de comprensión de lectura en el aula de español como segunda lengua o lengua extranjera. Filología y Lingüística, 41, 113125. https://doi.org/10.15517/rfl.v41i1.23743

Mato Vázquez, D., Espiñeira, E. y López Chao, V. A. (2017). Impacto del uso de estrategias metacognitivas en la enseñanza de las matemáticas. Perfiles Educativos, 39(158), 91 111. https://doi.org/10.22201/iisue.24486167e.2017.158.58759

Mengual García, E. Z. (2017). Metacomprensión e inteligencia emocional: relación e influencia en la comprensión lectora en alumnado de $5^{\circ}$ y $6^{\circ}$ de educación primaria (Tesis doctoral). Universidad Complutense de Madrid, Madrid, España. https://eprints.ucm.es/43467/1/T38947.pdf

Meniado, J. C. (2016). Metacognitive Reading Strategies, Motivation, and Reading Comprehension Performance of Saudi EFL Students. English Language Teaching, 9(3), 117-129. http://doi.org/10.5539/elt.v9n3p117

Munayco Medina, A. (2018). Influencia de los organizadores gráficos en la comprensión lectora de textos expositivos y argumentativos. Comuni@cción, 9(1), 5-13. http://www.scielo.org.pe/scielo.php?script=sci_arttext\&pid=S221971682018000100001

Muñoz Muñoz, Á. E. y Ocaña de Castro, M. (2017). Uso de estrategias metacognitivas para la comprensión textual. Cuadernos de Lingüística Hispánica, (29), 223-244. https://doi.org/10.19053/0121053X.n29.2017.5865

Muñoz, C., Valenzuela, J., Avendaño, C. y Núñez, C. (2016). Mejora en la motivación por la Lectura Académica: la mirada de estudiantes motivados. Ocnos: Revista de Estudios Sobre Lectura, 15(1), 52-68. https://doi.org/10.18239/ocnos_2016.15.1.941 
Nasab, M. S. B. y Motlagh, S. F. P. (2015). A complete review for metacognitive, cognitive, and social/affective strategies as essential components of learning strategies and their relationships with EFL learners' reading comprehension promotion. Advances in Language and Literary Studies, 6(3), 166-184. https://doi.org/10.7575/aiac.alls.v.6n.3p.166

Navarro, M., Orellana, P. y Baldwin, P. (2018). Validación de la Escala de Motivación Lectora en estudiantes chilenos de enseñanza básica. Psykhe, 27(1), 1-17. https://doi.org/10.7764/psykhe.27.1.1078

Neira Martínez, A. C., Reyes Reyes, F. T. y Riffo Ocares, B. E. (2015). Experiencia académica y estrategias de comprensión lectora en estudiantes universitarios de primer año. Literatura y Lingüística, 31, 221-244. https://doi.org/10.4067/S071658112015000100012

Núñez Benavidez, G.I. (2016). Aprendizaje de competencias versus aprendizaje de contenidos científicos. La competencia científica de explicar fenómenos científicamente relacionados con la estructura de la materia. Granada: Universidad de Granada. [http://hdl.handle.net/10481/43560]

$\begin{array}{llll}\text { OECD. (2019). PISA results. } & \end{array}$ http://www.oecd.org/pisa/Combined_Executive_Summaries_PISA_2018.pdf

Orbegoso G., A. (2016). La motivación intrínseca según Ryan \& Deci y algunas recomendaciones para maestros. Lumen: Educare, 2(1), 75-93. https://doi.org/10.19141/2447-5432/lumen.v2.n1.p.75-93

Osorio Sánchez, A., Mendoza Vargas, E. y Ballesteros Ballesteros, E. (2018). Importancia de la lectura en el desarrollo de las habilidades investigativas del estudiante universitario. Ciencia sociales y económicas, 2(1),71-91. https://doi.org/10.18779/csye.v2i1.267

Paba Barbosa, C. y González Sanjuán, R. (2014). La actividad metacognitiva y la comprensión lectora en estudiantes de décimo grado. Psicología Desde el Caribe, 31(1),

79-102. http://rcientificas.uninorte.edu.co/index.php/psicologia/article/view/5185

Ramírez Peña, P., Rossel Ramírez, K. y Nazar Carter, G. (2015). Comprensión lectora y metacognición: Análisis de las actividades de lectura en dos textos de estudio de la asignatura de lenguaje y comunicación de séptimo año básico. Estudios Pedagógicos, 41(2), 213-231. https://doi.org/10.4067/S0718-07052015000200013

Ruvalcabar Estrada, O. y Rodríguez Gómez, J. (noviembre 2019). Las estrategias metacognitivas y la motivación de logro asociadas con la comprensión lectora. [Sesión de Congreso]. XV Congreso nacional de investigación educativa, Acapulco, México. http://www.comie.org.mx/congreso/memoriaelectronica/v15/doc/0958.p 
Sánchez, H., Reyes, C. y Mejía, K. (2018). Manual de términos en investigación científica, tecnológica y humanística. Lima: Universidad Ricardo Palma. http://repositorio.urp.edu.pe/handle/URP/1480

Schunk, D. H. (2012). Teorías del aprendizaje. Una perspectiva educativa (6ta ed.). Pearson Educación.

Sedano Fernández, M. (2015). Leer en el aula: propuesta para mejorar la lectura en secundaria. Opción, 31(6), 1136-1159. https://produccioncientificaluz.org/index.php/opcion/article/view/20789/20668

UNESCO. (2017). Más de la mitad de los niños y adolescentes en el mundo no está aprendiendo. Ficha informativa 46, 1-25. http://uis.unesco.org/sites/default/files/documents/fs46-more-than-half-children-notlearning-2017-sp.pdf

Valenzuela, M. Á. (2019). ¿Qué hay de nuevo en la metacognición? Revisión del concepto, sus componentes y términos afines. Educação e Pesquisa, 45(39), 1-20. https://doi.org/10.1590/S1678-4634201945187571 